\title{
Performances of the UNDERground SEISmic array for the analysis of seismicity in Central Italy
}

\author{
Gilberto Saccorotti $\left({ }^{1}\right)$, Bellina Di Lieto $\left({ }^{2}\right)$, Fabrizio Tronca $\left({ }^{1}\right)$, Costantino Fischione $\left({ }^{1}\right)$, \\ Roberto Scarpa $\left({ }^{2}\right)$ and Rosanna Muscente $\left.{ }^{3}\right)$ \\ ${ }^{(1)}$ Istituto Nazionale di Geofisica e Vulcanologia, Osservatorio Vesuviano, Napoli, Italy \\ (2) Dipartimento di Fisica «E.R. Caianello», Università degli Studi di Salerno, Baronissi (SA), Italy \\ (') Parco Scientifico e Tecnologico d'Abruzzo, L'Aquila, Italy
}

\begin{abstract}
This paper presents the first results from the operation of a dense seismic array deployed in the underground Physics Laboratories at Gran Sasso (Central Italy). The array consists of 13 short-period, three-component seismometers with an aperture of about $550 \mathrm{~m}$ and average sensor spacing of $90 \mathrm{~m}$. The reduced sensor spacing, joined to the spatially-white character of the background noise allows for quick and reliable detection of coherent wavefront arrivals even under very poor SNR conditions. We apply high-resolution frequency-slowness and polarization analyses to a set of 27 earthquakes recorded between November, 2002, and September, 2003, at epicentral distances spanning the $20-140 \mathrm{~km}$ interval. We locate these events using inversion of $P$ - and $S$-wave backazimuths and $S$ - $P$ delay times, and compare the results with data from the Centralized National Seismic Network catalog. For the case of $S$-wave, the discrepancies among the two set of locations never exceed $10 \mathrm{~km}$; the largest errors are instead observed for the case of $P$-waves. This observation may be due to the fact that the small array aperture does not allow for robust assessment of waves propagating at high apparent velocities. This information is discussed with special reference to the directions of future studies aimed at elucidating the location of seismogenetic structures in Central Italy from extended analysis of the micro-seismicity.
\end{abstract}

Key words seismic array - microearthquakes Central Apennines

\section{Introduction}

Over the past 40 years, much attention has been given to the use of instrumental arrays to detect plane-wave signals in seismological time series and to measure wave slowness vectors (Rost and Thomas, 2002; Schweitzer et al.,

Mailing address: Dr. Gilberto Saccorotti, Istituto Nazionale di Geofisica e Vulcanologia, Osservatorio Vesuviano, Via Diocleziano 328, 80124 Napoli, Italy; e-mail: gilberto@ov.ingv.it
2002). Compared to classical seismic networks, the main advantage of seismic arrays consists in their ability to detect weak or emergent signals, and to allow for an effective noise reduction through multichannel waveform stacking. Moreover, once combined with data from adjacent networks, the wave azimuths determined at one or several arrays provide strong constraints on the location of low-magnitude events detected by a small number of stations (e.g., Bratt and Bache, 1985). Such reduction in the detection threshold represents a critical step toward a successful assessment of the location, geometry and kinematics of active fault systems.

Following these arguments, the University of Salerno, the Italian National Institute for $\mathrm{Nu}-$ clear Physics and National Institute for Geo- 
physics and Volcanology (hereinafter referred to as INFN and INGV, respectively) conducted a joint effort for the deployment of a smallaperture, dense array of short period seismometers in the Gran Sasso (Central Italy) underground Physics Laboratories. Designed and tested during the late 90's, the underground array (UNDERSEIS) became fully operative by early May 2002, providing low-noise, high-dynamic continuous recordings of ground shaking signals (Scarpa et al., 2004). In Italy, multichannel deployments have already been successfully tested for their detection capabilities on local seismicity (e.g., Braun et al., 2004); however, UNDERSEIS represents the first example of a seismic antenna deployed in an underground setting. This paper presents the first results from the operation of the UNDERSEIS array. We first describe the array configuration and setting, and discuss its resolving capabilities in detecting and measuring plane-wave signals. We then describe the techniques routinely used for retrieving the propagation and polarization parameters from the multichannel recordings. Examples of application are presented for a set of low magnitude $\left(M_{d}<3\right)$ earthquakes recorded at local and regional distances. $S$ - $P$ delay times and backazimuths of these events are then used to obtain epicentral estimates, the robustness of which is demonstrated by comparison with the catalogue locations from the INGV's Centralised National Seismic Network (CNSN). These results will be discussed further with special reference to the directions for future works aimed at a) improving the array performance, and b) investigating the details of wave propagation and the location of low-magnitude events in the investigated area.

\section{The UNDERSEIS arrey}

Located in Central Italy, some $120 \mathrm{~km}$ ENE of Rome, the Gran Sasso is a limestone massif

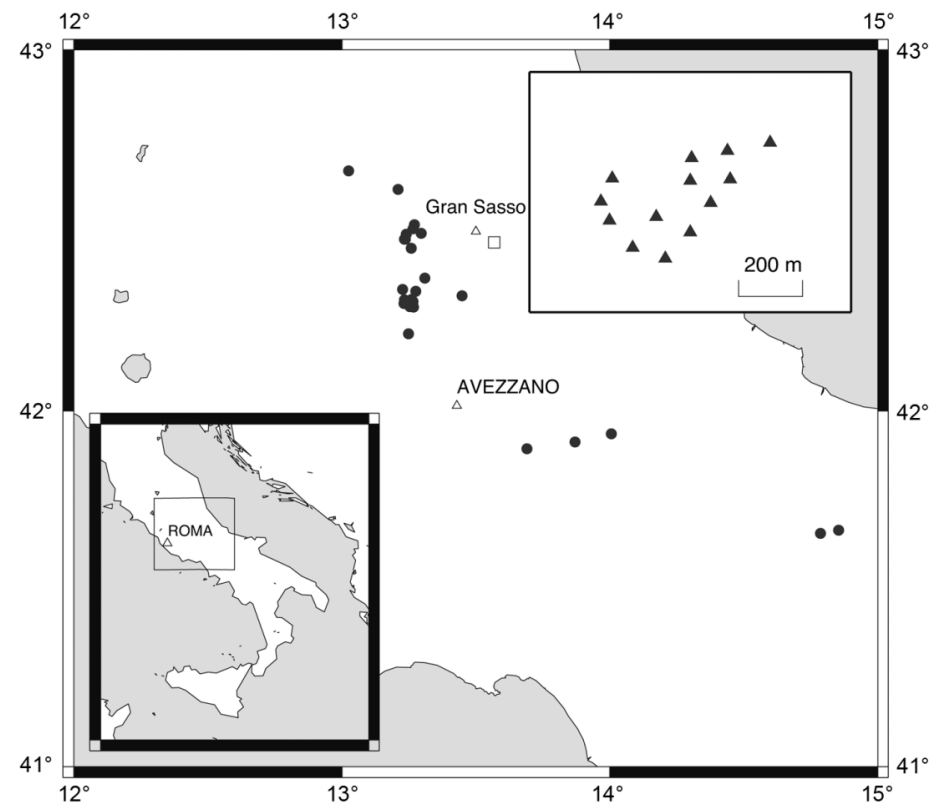

Fig. 1. Map of Central Italy with location of the Gran Sasso INFN laboratories (square) and epicenters of the analysed earthquakes (black dots). Sites mentioned in the text are labeled. The inset at the upper rigth shows the configuration of the UNDERSEIS seismic array. 

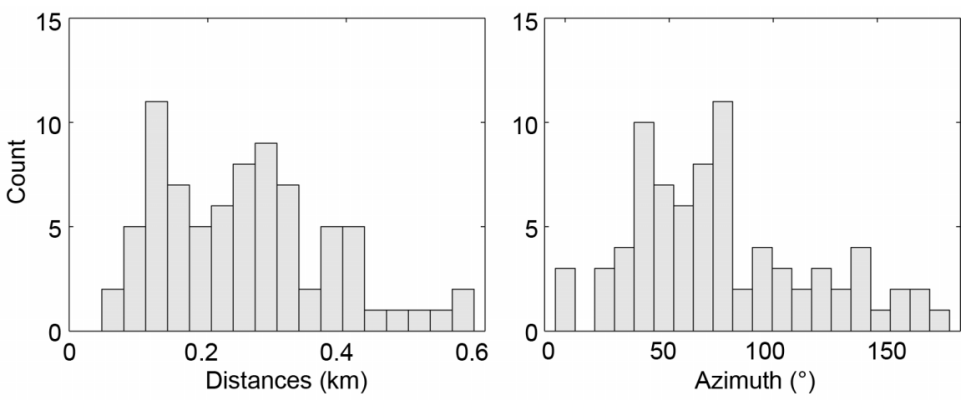

Fig. 2. Histograms of station spacing (left) and relative orientation (right) for all the indepedent station pairs of the array.

constituting the highest peak $(2914 \mathrm{~m})$ of the Apennines Chain (fig. 1). Since 1986, subnuclear particle physics laboratories have been hosted in underground tunnel systems located at a mean elevation of $1500 \mathrm{~m}$, being therefore naturally shielded by a 1400 -m-thick limestone cap. The area is affected by major seismicity associated with the mainly distensive tectonics affecting the Apennines since the Late Pliocene. The major historical event was the 1915, $M_{s}=6.8$ Avezzano earthquake, which caused about 32000 casualties (Amoruso et al., 1998). In addition, low-to moderate-size earthquakes occur quite frequently, the most recent in 1992, 1994 and 1996 (De Luca et al., 2000; Chiarabba et al., 2005). In the same area a temporary, large-scale seismic deployment was installed aimed at defining the location and geometry of seismogenic structures from microseismicity data (Chiarabba et al., 2004).

The low noise associated with the underground setting (De Luca et al., 1998), and the quite considerable seismicity make the Gran Sasso labs an ideal site for high resolution seismic observations. The design of the UNDERSEIS hardware and software components began on the late 90's; the array became fully operative by May, 2002. UNDERSEIS intersects a main seismogenetic fault where the occurrence of slow earthquakes has recently been detected through two wide-band geodetic laser interferometers (Crescentini et al., 1999; Amoruso et al., 2002). In its present configuration, the array consists of 13 elements, each equipped with a MARK Prod- uct L4C-3D, $1 \mathrm{~Hz}$ 3-component seismometer, whose signals are digitized at a sampling interval of $0.01 \mathrm{~s}$ and a dynamic range of 24 bits. Further details on the design and working principles of the array are reported in Scarpa et al. (2004).

In its present configuration, UNDERSEIS depicts an average sensor spacing of about $90 \mathrm{~m}$ and the widest distance among sensors of about $550 \mathrm{~m}$ (fig. 1). Location of the array elements was constrained by the geometry of the tunnel system, thus hindering the achievement of uniform station spacing and relative azimuths distribution which are warranted for multichannel analyses (fig. 2). Recently, however, new galleries have been made available, and installation of additional array elements is already in progress with the specific goal of improving array aperture and geometry.

\section{Array performance}

In this section we investigate the array's capabilities and limitations, in turn deriving useful hints for the selection of parameters to be adopted for the subsequent frequency-slowness measurements.

\subsection{Array response patterns}

The resolving capabilities of a multichannel system are conveniently represented by the «Beam Pattern» function (Capon, 1969) 


$$
B(\vec{S})=\frac{1}{N^{2}}\left|\sum_{i}^{N} e^{-\vec{k} \cdot\left(\overrightarrow{x_{i}}-\vec{x}_{0}\right)}\right|^{2}
$$

where $|K|=2 \pi / \lambda$ is the wave number corresponding to wavelength $\lambda, N$ is the number of stations, the $\vec{x}_{i} \mathrm{~S}$ are the station coordinates, and $\vec{x}_{0}$ is the vector position of the reference sensor. This function describes the shape of the broad-band wave number spectrum for a vertically-incident plane wave. In practice, it represents the 'pulse response' of the array in the wavenumber domain, and is particularly useful for investigating the effects of spatial aliasing which manifest as spurious peaks located at wave-numbers other than the origin. The UNDERSEIS wavenumber spectrum is shown in fig. 3. As a consequence of the ENEWSW elongation of the array, the main spectral peak depicts a marked NNW-SSE trend, this implying low resolving capabilites once measuring waves propagating along that particular direction.

Aliasing peaks appear at wavenumbers of abouts 20 cycles $/ \mathrm{km}$, corresponding to wavelengths in the order of $300 \mathrm{~m}$. On the other side,

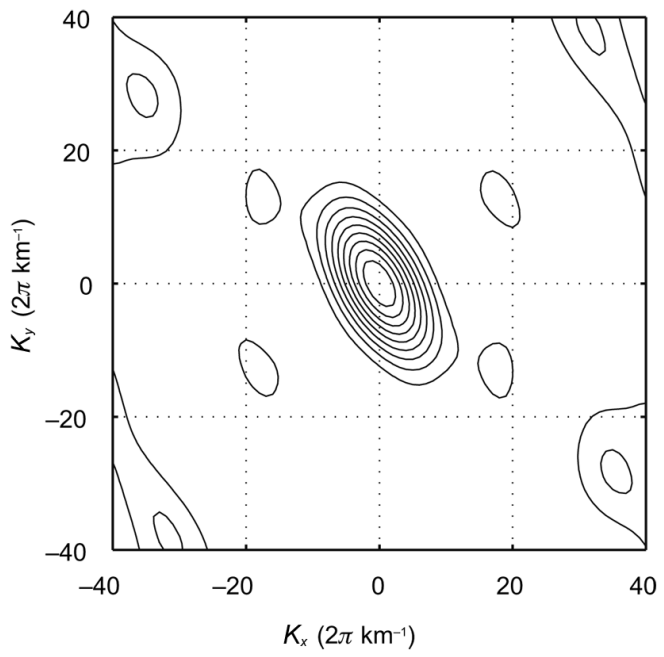

Fig. 3. Broad-band beam pattern spectrum (see eq. (3.1) in text) representing the response of the array to a monochromatic, vertically-inciding plane wave. $K_{x}$ and $K_{y}$ are the two components of the wavenumber vector with respect to a Cartesian system in which the $x$ - and $y$-axes are oriented W-E and S-N, respectively. the lowest bounds for body waves apparent velocities correspond to the seismic velocities of the shallowest crust, here taken equal to $4 \mathrm{~km} / \mathrm{s}(P$ waves) and $2.3 \mathrm{~km} / \mathrm{s}$ ( $S$-waves). Consequently, the Nyquist frequencies for the two wavetypes are in the order of $\approx 13 \mathrm{~Hz}$ and $\approx 6 \mathrm{~Hz}$, respectively.

\subsection{Signal coherency}

All multichannel processing methods are based upon the common waveform model of the signal: in other words, it is assumed that the signal recorded by each array element is the same except for a phase factor associated with the propagation across the deployment. In order to fullfil such assumption, it is crucial for the signal to maintain significant coherency throughout the different sensors. Using a number of $P$ - and $S$ wave arrivals recorded at different SNRs, we calculated the array-averaged signal coherency as a function of frequency for both type of waves using the formula (Saccorotti and Del Pezzo, 2000):

$$
C(f)=\operatorname{atanh}\left(\frac{1}{N_{p}} \sum_{i, j}^{N} c z_{i, j}\right) \quad i \neq j
$$

where $f$ is the frequency, $N_{p}=N(N-1) / 2$ is the number of independent station pairs in the array, and $c z_{i, j}$ is the Fisher's $Z$-transform of the correlation coefficient among the band-pass filtered signals from stations $i$ and $j$.

Signal coherency is expected to vary from event to event as a function of a number of factors, including SNR and the frequency content of the signal and noise. However, we observed that in general $P$-waves maintain significant correlations over the $1-10 \mathrm{~Hz}$ frequency range, while $S$-wave are significantly coherent only over the $1-6 \mathrm{~Hz}$ frequency range. Combined with the Nyquist frequency limits for spatial aliasing mentioned above, these considerations provided constraints on the frequency bands to adopt for the analysis of earthquake wavefields described throughout the next sections. Similar correlation analyses were performed over different noise windows collected at day- and nigh-times. For the whole set of interstation distances, significant noise coherency was only observed in association with marine microseisms, which typically manifest at frequencies 

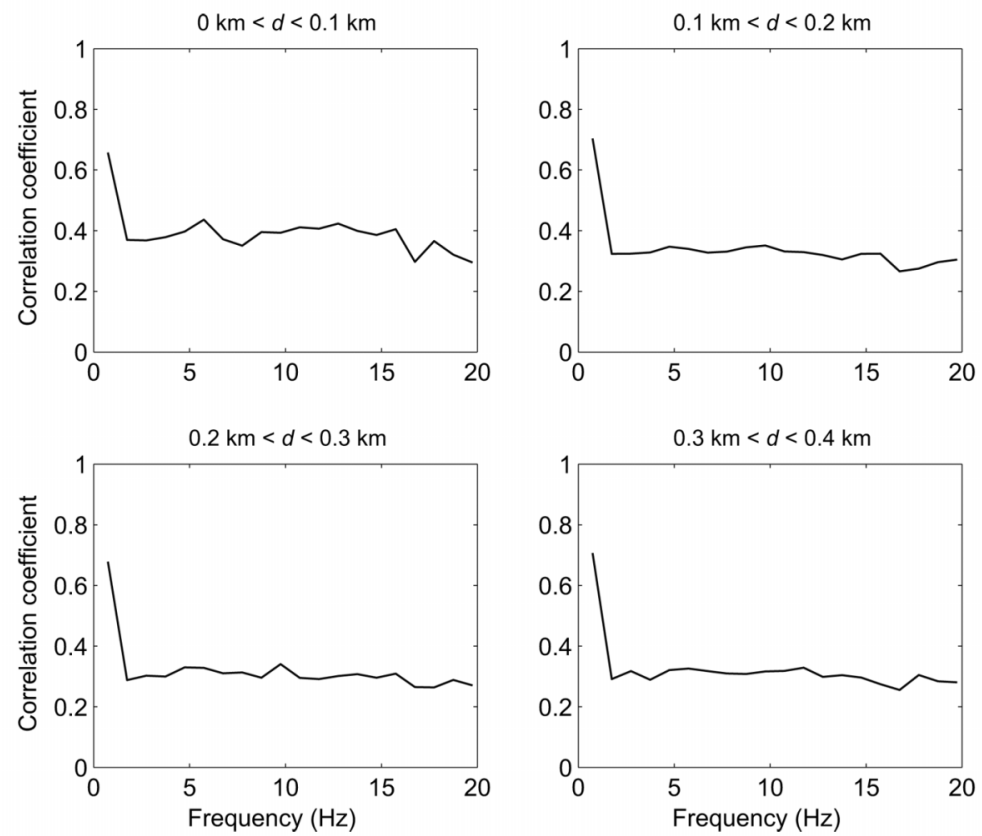

Fig. 4. Noise correlation as a function of frequency for different distance ranges. The correlation functions have been obtained from averaging the frequency-dependent correlation estimates associated with station pairs whose spacing $\mathrm{d}$ is included into the bounds reported at the top of each plot.

lower than $\approx 1 \mathrm{~Hz}$ (fig. 4). At higher frequencies, the correlation plots reported in fig. 4 fully support the assumption of spatially-uncorrelated seismic noise required by the multichannel processing schemes presented in the following.

\section{Methods of analysis}

We analysed UNDERSEIS data using upto-date techniques aimed at fully exploiting the resolving capabilities of a multi-channel system toward measurement of the propagation and polarization parameters of the incident wavefields.

\subsection{Polarization}

For polarization analysis, we adopt the technique proposed by Jurkevics (1988), which consists of a multichannel extension of the original covariance method of Kanasewich (1981). For a given signal window, the polarization parameters (azimuth and incidence angles, degree of linearity) are derived from the eigenvectors and relationships among eigenvalues of an array-averaged covariance matrix of the three-components of ground motion. In theory, the array-averaging procedure allows the contribution of noise (here assumed to be spatially uncorrelated) to be reduced by a factor $\sqrt{N}$ to the covariance estimates. For each window of analysis, signals from individual stations should be aligned according to the slowness measured for that window. In our situation, however, alignment is not necessary considering that the inter-station time delays are much smaller than the dominant period of the signal.

\subsection{Frequency-slowness analysis}

For extracting the propagation parameters of UNDERSEIS data we use MUSIC, the MUltiple SIgnal Classification technique (Schmidt, 


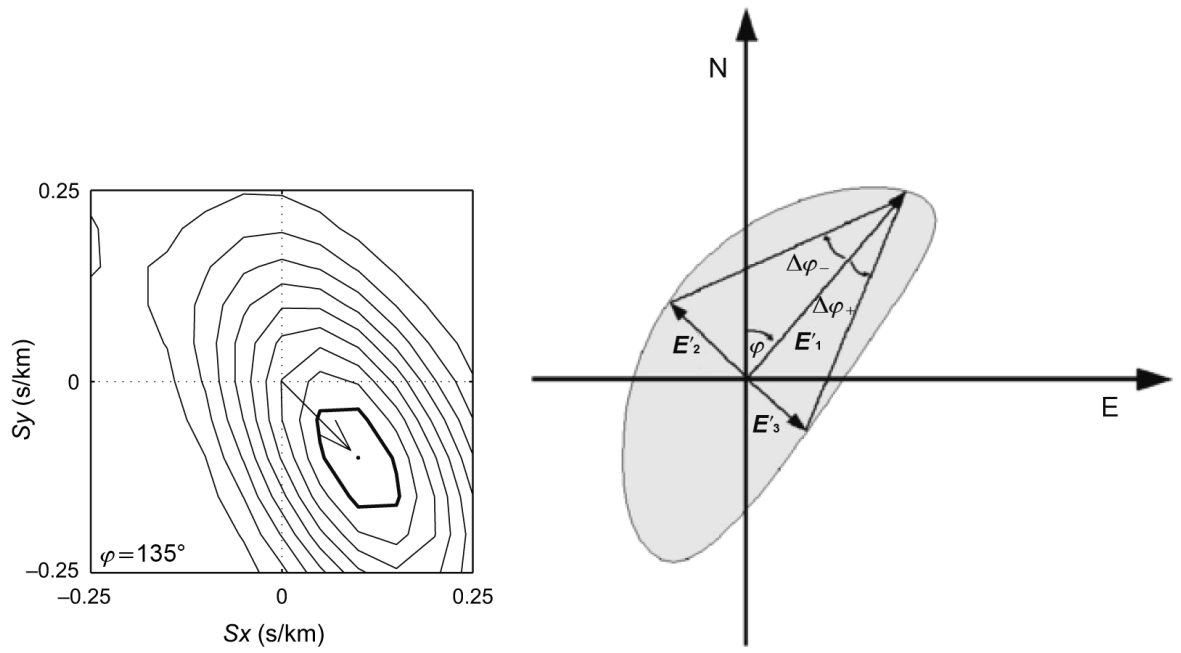

Fig. 5. Sketch of the procedures used for retrieving uncertainties in the estimates of the propagation (left) and polarization (right) parameters. On the left, a sample slowness spectrum is displayed, with evidenced the contour line encompassing that region for which the power is greater than $90 \%$ of the main peak's power. This region is used to define errors in azimuth and ray parameter estimates. On the right plot, the shaded area marks the projection onto the horizontal plane of the polarization ellipsoid. $E^{\prime}$ are the horizontal projections of the three eigenvectors; $\varphi$ is the estimated polarization azimuths, whose uncertainties are defined through the angles $\Delta \varphi$.

1986; Goldstein and Archuleta, 1987, 1991). Once compared to more classical methods (e.g., Beamforming or Capon's (1969) High Resolution), MUSIC has demonstrated superior performances for both the cases of low SNR signals and more plane waves simultaneously impinging on the array (e.g., Goldstein and Archuleta, 1987, 1991; Chiou and Bolt, 1993). For a given window of analysis, the MUSIC slowness spectrum is expressed as (Chiou and Bolt, 1993)

$$
Q(\vec{S})=\frac{1}{1-\sum_{i=1}^{M}\left|A(\vec{S})^{H} \cdot V_{i}\right|^{2}}
$$

where $M$ is the number of signals impinging at the array, whose number is estimated applying Akaike's information Criterium (AIC; Wang and Kaveh, 1985) to the ordered set of eigenvalues of the spatial Cross-Spectral Matrix (CSM); $V_{i}$ is the $i$-th eigenvector of the CSM, and $A(\vec{S})^{H}$ is the Hermitian of a column vector containing the expected inter-station phase delays for a monochromatic plane-wave of frequency $\omega_{0}$ propagating with slowness $\vec{S}$. Note that the ele- ments of vector $\boldsymbol{A}$ correspond to the terms in the summation of eq. (3.1) above. Maximizing eq. (3.2) means finding those slowness vector(s) for which the array response has a maximum projection onto the signal subspace, which is spanned by the $i=1, \ldots, M$ eigenvectors $V_{i}$. Peaks in the $Q(\vec{S})$ function above are thus associated with the horizontal slownesses of individual plane-wave components crossing the array. The amplitude of these peaks gives a measure of the extent to which the observed inter-station phase delays fit the model of a plane-wave propagating at the particular slowness $\vec{S}$, and is completely independe of the actual amplitude of the signal.

\subsection{Uncertainties estimation}

We obtain an empirical estimate of the uncertainties associated with the measurement of propagation parameters by considering the range of horizontal slownesses and propagation azimuths associated with points of the slowness spectrum whose power is greater than $90 \%$ of 
the peak's power (fig. 5, left). For the polarization azimuth, angular uncertainties are derived from the ratio of the horizontal projection of the principal and secondary eigenvectors (fig. 5, right). This procedure represents a first attempt toward accounting for the large azimuthal uncertainties which arise for small incidence angles, even when the motion is very rectilinear. A similar procedure is followed for obtaining the uncertainties on the incidence angle.

\section{Analysis of local and regional seismicity}

Figure $6 \mathrm{a}, \mathrm{b}$ shows sample seismograms and amplitude spectra for the vertical-and transverse-
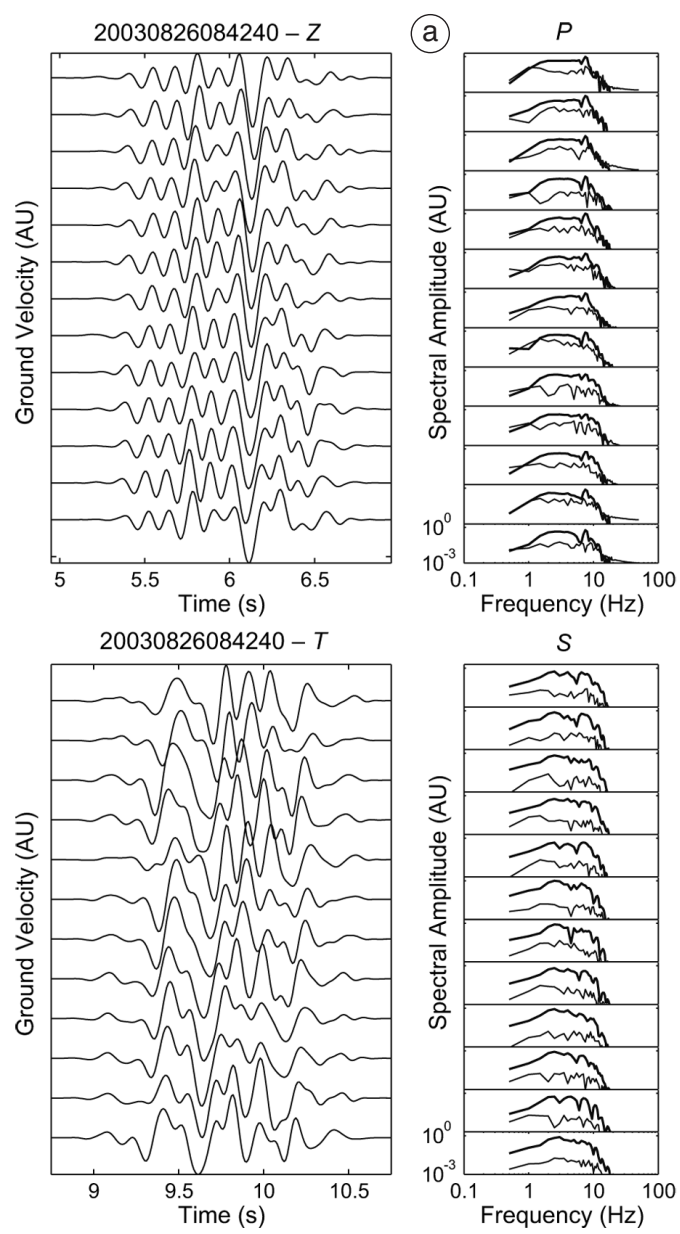
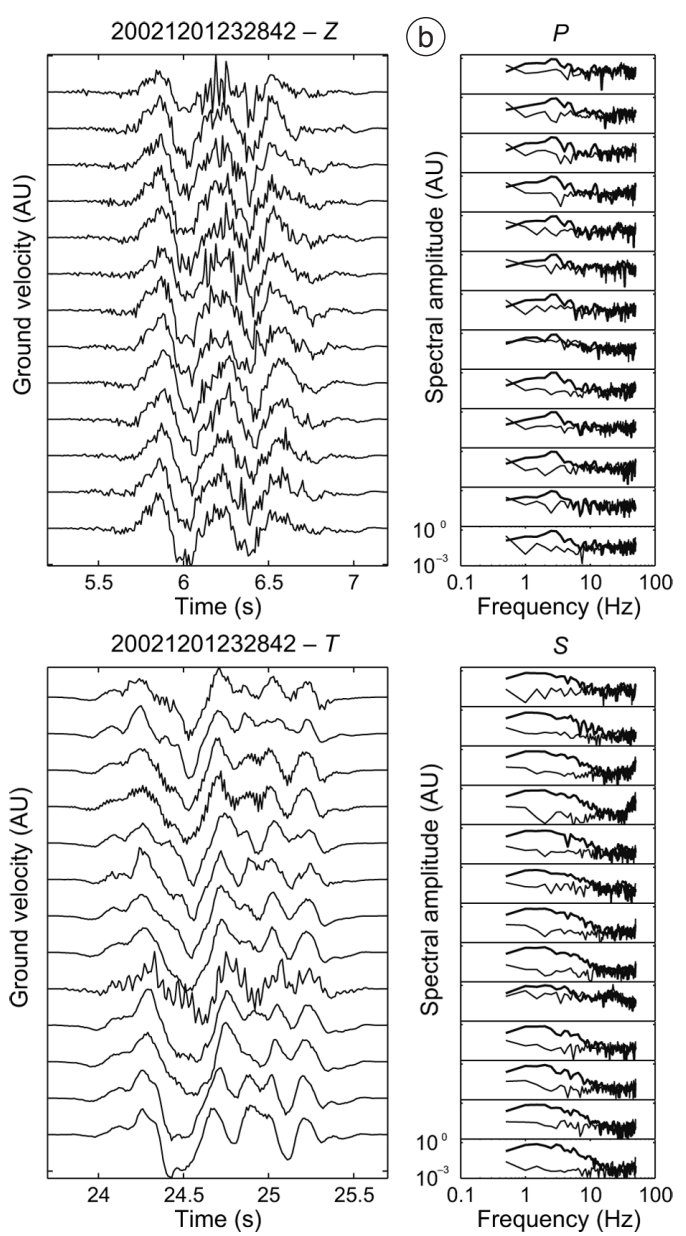

Fig. 6a,b. a) Raw velocity seismograms and amplitude spectra for the $P$ - and $S$-wave arrivals as recorded by the vertical-and rotated transverse-component, respectively, for a $M_{d}=2.4$ earthquake on August 26, 2003, at 08:43:56 GMT. The epicentral range is about $25 \mathrm{~km}$. Seismograms and spectra are normalized to the largest-amplitude trace. Thin lines in the spectral plots refer to noise spectra calculated over 2-s-long windows preceeding the onset of the event. b) The same as in a), but for a $M_{d}=3.3$ earthquake on December 1, 2002, at 23:29:23 GMT. The epicentral range is about $124 \mathrm{~km}$. 
component of ground velocity associated with two earthquakes recorded at local and regional distances. For both cases, the figure also reports the amplitude spectra associated with noise windows preceeding the onset of the events. Despite the low magnitude of these earthquakes, seismograms depict an excellent Signal-to-Noise Ratio, and significant wave coherence is observed among the different array elements. In the following, we proceed by describing application of the techniques illustrated above to an heterogenous set of earthquakes as recorded by UNDERSEIS.

The first step in calculating MUSIC slowness spectra is to obtain robust estimates of the narrow-band spatial covariance of the signal. There- fore, we first band-pass-filter the array recordings over a narrow frequency band using a zero-phaseshift, 2-pole Butterworth filter. The filtered signals are then Hilbert-transformed, and converted to Analytic Signals which eventually serve to obtain the complex-valued estimates of the spatial covariance. Eigenvalues and eigenvectors of the covariance matrix are derived using Singular Value Decomposition. In order to account for the variable frequency content associated with different wavetypes, we conduct the analysis over consecutive, 2-Hz-wide frequency bands spanning the $1-10 \mathrm{~Hz}$ and $1-6 \mathrm{~Hz}$ frequency range for $P-$ and $S$-waves, respectively. Slowness spectra evaluated over individual frequency bands are then

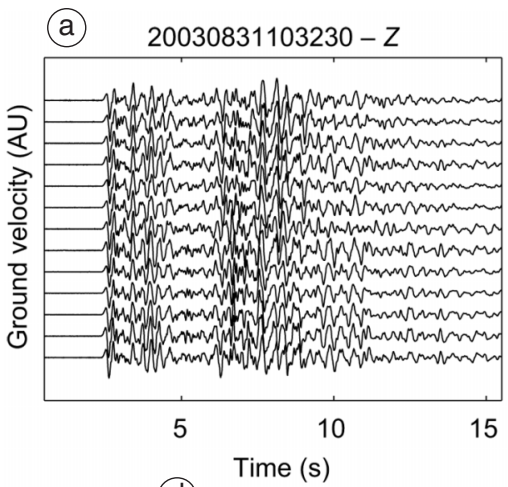

(b)

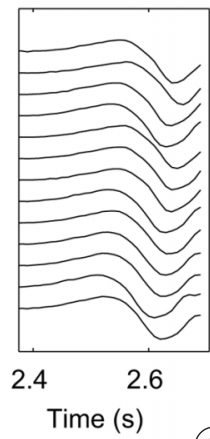

Time (s) (c)

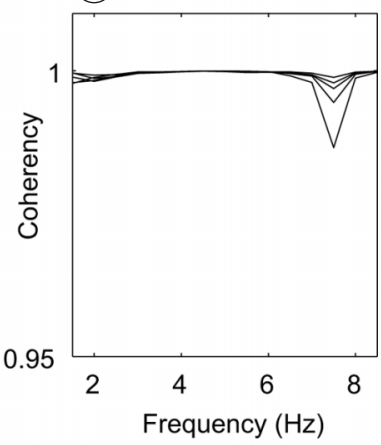

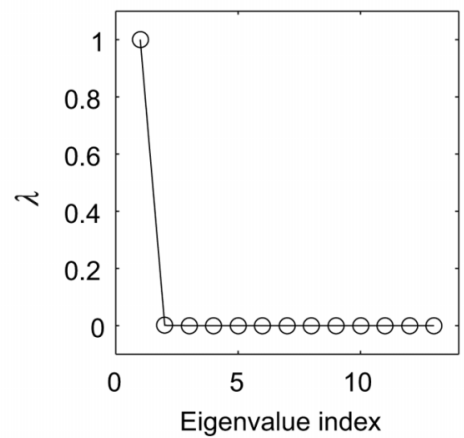

Fig. 7a-e. a) Vertical-component seismograms for an event occurred at 10:32:30 GMT on August 31, 2003. b) Time interval used for $P$-wave slowness analysis. c) Multichannel signal Coherency (eq. (3.2) in text) over the frequency band selected for the analysis. The different lines refer to the five consecutive time position of the window of analysis. d) The ordered set of normalized eigenvalues derived from diagonalization of the spatial covariance matrix. e) Time-Frequency stacked slowness spectrum. Propagation azimuth $\phi$ and ray parameter $|P|$ associated with the peak value are reported at the bottom left of the plot. The arrow points to the direction of wave propagation. 


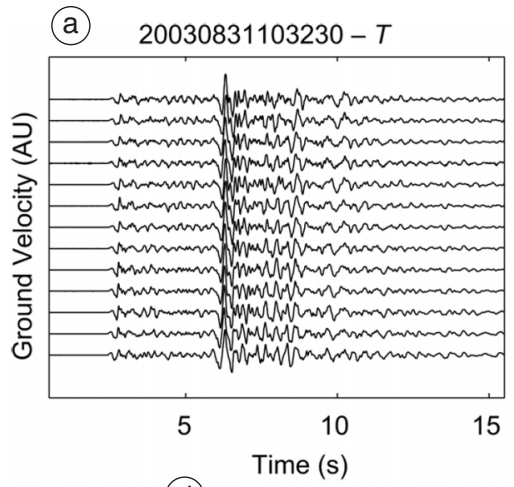

(b)

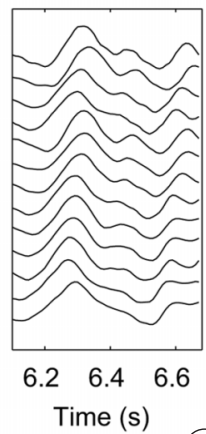

Time (s)

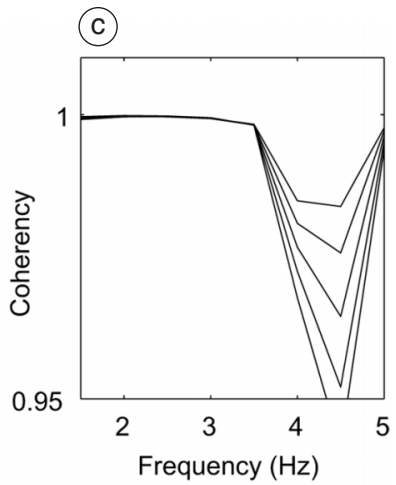

(e)
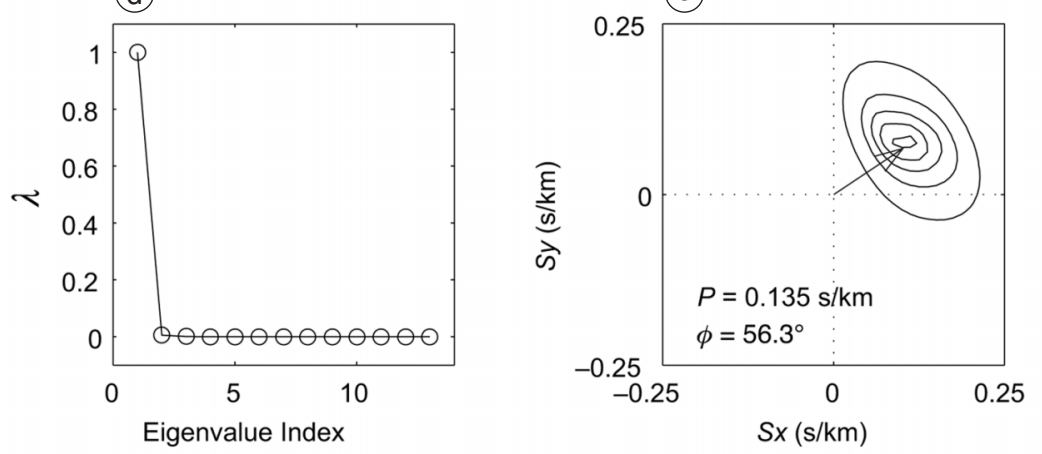

Fig. 8a-e. The same as in fig. 7a-e, but for the $S$-wave arrival measured on the transverse components.

stacked to obtain a broad-band spectrum in which the contribution of body-waves is enhanced and the effects of the frequency-dependent peaks due to either spatial aliasing or surface-wave contaminations is instead attenuated (Spudich and Oppenheimer, 1986). This procedure is repeated over five consecutive time windows encompassing the selected wave arrival, and a final slowness spectrum is derived from the stack of these timevarying estimates. We first apply slowness analysis to $P$-wave arrivals recorded at the vertical components. Then, we rotate the horizontal components into the transverse direction to the backazimuth obtained from the $P$-wave, and evaluate $S$-wave slowness spectra over the rotated components.

Examples of application of MUSIC slowness measurements are displayed in figs. 7a-e and 8a-e, which report $P$ - and $S$-waves slow- ness spectra obtained for a $M_{d}=2.4$ earthquake recorded on August 31, 2003, at an epicentral distance of about $25 \mathrm{~km}$. Figure $9 \mathrm{a}-\mathrm{f}$ depicts the particle motion patterns for the $P$ - and $S$-wave arrivals of the same event.

In addition to the slowness spectra and coherence plots associated with the selected time windows, figs. 7a-e and 8a-e also report the ordered set of eigenvalues derived from diagonalization of the spatial covariance matrix. The absolute dominance of the main eigenvalue over the remaining ones indicates that a single signal is impinging on the array, in turn confirming the marked separation between the signal and noise subspaces previously postulated on the basis of noise correlation analyses.

The performances of UNDERSEIS toward detection and location of local seismicity become even more evident for the case of low 
(a)

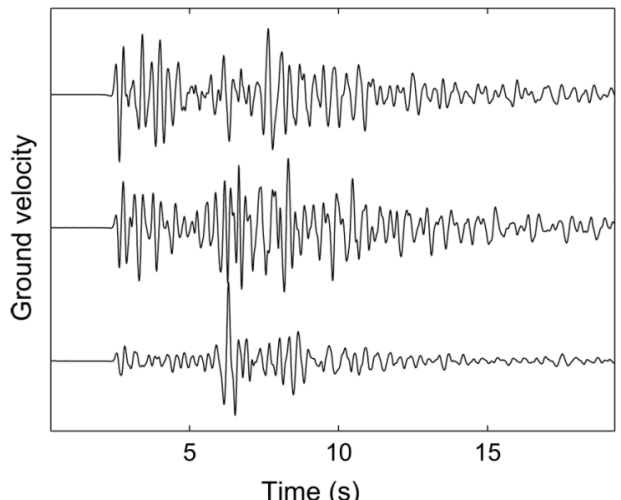

(d)

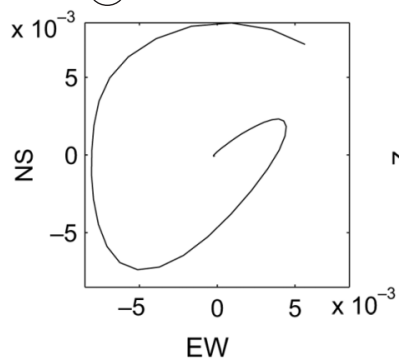

(b)

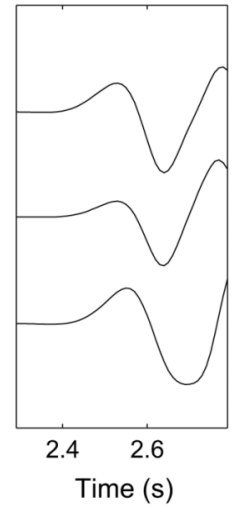

(C)

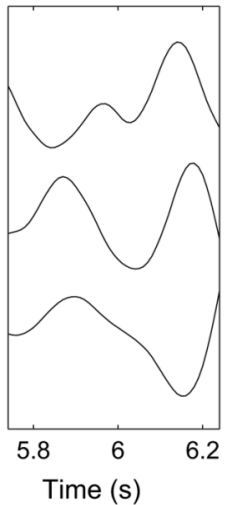

(f)
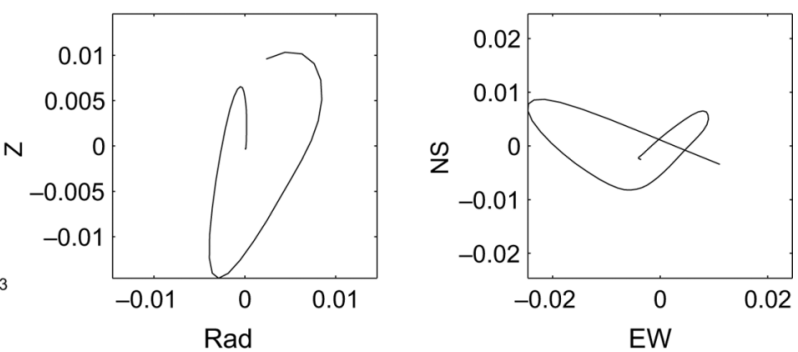

Fig. 9a-f. a) Three-component, array-stacked waveforms for the same event displayed in figs. 7a-e and 8a-e. b, c) Time windows respectively used for $P$ - and $S$-wave polarization estimates. d, e) $P$-wave particle motion orbits over the horizontal and radial-vertical plane, respectively. f) $S$-wave particle motion over the horizontal plane. $P$-wave polarization azimuth and incidence angles obtained from the covariance analysis are $35^{\circ}$ and $12^{\circ}$, respectively. The $S$-wave polarization azimuth is $110^{\circ}$.

SNR signals. An example is given by a $M_{d}=2$ earthquake which occurred on December 27, 2002 , and located by the RSNC at coordinates $41.938^{\circ} \mathrm{N}, 14.007^{\circ} \mathrm{E}, \mathrm{ESE}$ of the town of Sulmona. This event was recorded by UNDERSEIS with a very low amplitude, thus making impossible any visual estimate of the $P$-wave onset (fig. 10). However, the multichannel analyses provide consistent slowness estimates, which eventually permit a robust location of this event.

For the sample earthquake reported in fig. 10 , both the power of the stacked slowness spectrum and the multichannel coherency before the signal's onset are very low, thus confirming that the background noise is essentially uncorrelated among the different array el- ements. Based on this property, the power of the slowness spectra may be used as a powerful tool for automatic signal discrimination applied to real-time, routine analysis.

The above procedures are extended to a further 25 earthquakes between November 2002 and September 2003. Magnitudes and epicentral distances of these events span the $2.3 \leq M \leq 3.5$ and $20-140 \mathrm{~km}$ ranges, respectively (table I). Figure 11 illustrates the relationship among the measured $P$ - and $S$-waves ray parameters and the epicentral range, here presented in terms of $S-P$ delay time. For the two farest events $(\Delta \approx 140 \mathrm{~km}), P$-wave ray parameters range between $0.1 \mathrm{~s} / \mathrm{km}$ and $0.15 \mathrm{~s} / \mathrm{km}$, corresponding to apparent velocities spanning the $6.5-10 \mathrm{~km} / \mathrm{s}$ range. Once accounting for the measurement 

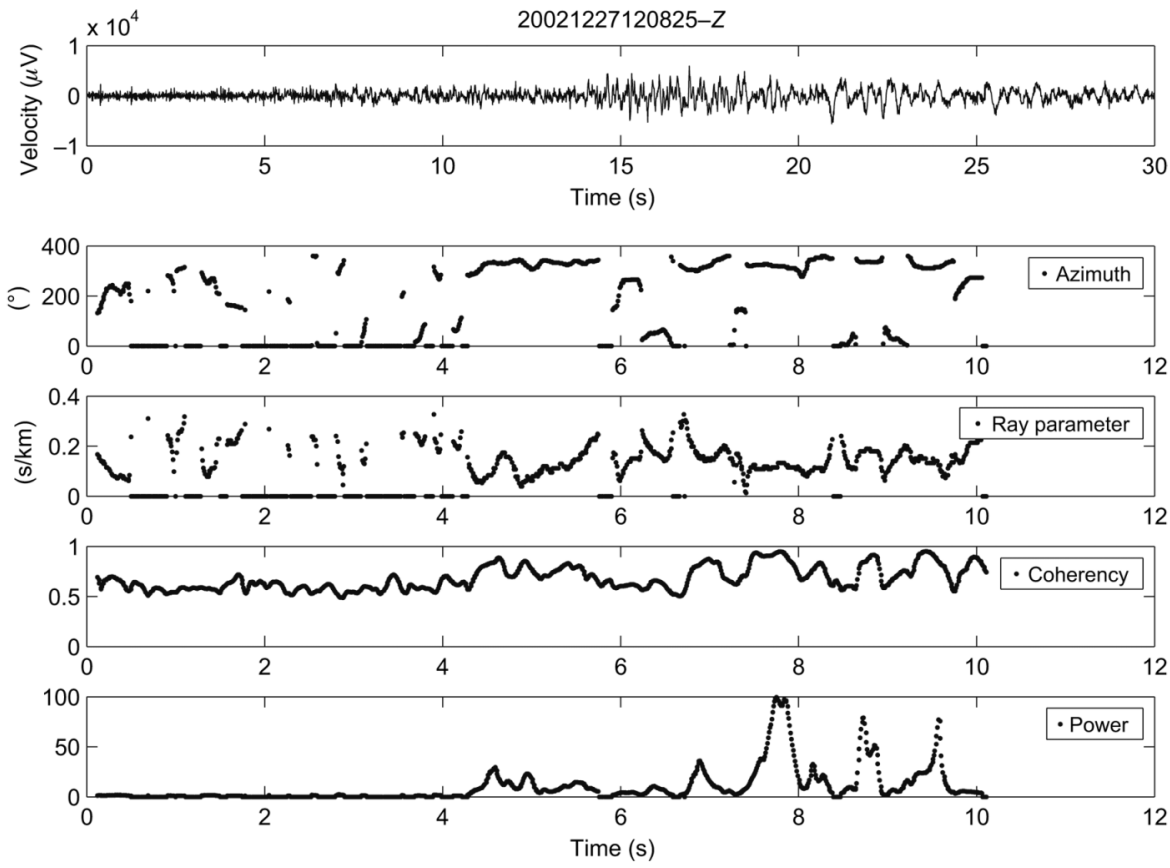

Fig. 10. Time behaviour of the propagation parameters for a $M_{d}=2$ event at 12:42 GMT on December 27, 2002, at an epicentral distance of about $80 \mathrm{~km}$. From top to bottom, sample vertical-component seismogram, propagation azimuth, ray parameter, multichannel coherency and power of the frequency-stacked slowness spectra. The $P$-wave arrival is marked by the sudden increase in both the slowness spectral power and coherency values about $4 \mathrm{~s}$ into the event.

Table I. List of analysed earthquakes.

\begin{tabular}{ccccccccc}
\hline \hline Event ID & Year & Month & Day & OT & $T s-T p(\mathrm{~s})$ & $M_{d}$ & RSNC Lat $\left(^{\circ}\right)$ & RSNC Long $\left(^{\circ}\right)$ \\
\hline 8 & 2002 & 11 & 20 & $02: 31: 51$ & 3.1 & 2.4 & 42.480 & 13.240 \\
9 & 2002 & 12 & 1 & $23: 29: 23$ & 16.5 & 3.3 & 41.660 & 14.790 \\
10 & 2002 & 12 & 2 & $20: 52: 59$ & 18 & 2.7 & 41.670 & 14.860 \\
12 & 2002 & 12 & 23 & $04: 17: 40$ & 5.5 & 2.3 & 42.688 & 13.027 \\
14 & 2002 & 12 & 24 & $07: 14: 05$ & 3.3 & 2.7 & 42.490 & 13.240 \\
15 & 2002 & 12 & 27 & $12: 42: 37$ & 7.3 & 3 & 41.940 & 14.010 \\
1 & 2003 & 1 & 1 & $20: 23: 40$ & 7.5 & 2.6 & 41.900 & 13.690 \\
3 & 2003 & 1 & 5 & $12: 41: 22$ & 2.7 & 3 & 42.500 & 13.300 \\
4 & 2003 & 4 & 7 & $15: 01: 15$ & 2.8 & 2.5 & 42.320 & 13.450 \\
5 & 2003 & 4 & 10 & $05: 50: 34$ & 7.5 & 2.5 & 41.920 & 13.870 \\
6 & 2003 & 4 & 11 & $11: 49: 10$ & 3 & 2.5 & 42.510 & 13.270 \\
7 & 2003 & 5 & 2 & $20: 14: 09$ & 2.8 & 2.6 & 42.450 & 13.260 \\
181 & 2003 & 6 & 30 & $22: 32: 20$ & 3.5 & 2.5 & 42.300 & 13.240 \\
186 & 2003 & 7 & 5 & $05: 07: 43$ & 3.3 & 3.1 & 42.310 & 13.270 \\
187 & 2003 & 7 & 6 & $09: 13: 40$ & 3.4 & 2.2 & 42.310 & 13.230 \\
\hline
\end{tabular}


Table I (continued).

\begin{tabular}{ccccccccc}
\hline \hline Event ID & Year & Month & Day & OT & $T s-T p(\mathrm{~s})$ & $M_{d}$ & RSNC Lat $\left(^{\circ}\right)$ & RSNC Long $\left(^{\circ}\right)$ \\
\hline 188 & 2003 & 7 & 7 & $12: 37: 12$ & 3.8 & 2.2 & 42.340 & 13.230 \\
192 & 2003 & 7 & 11 & $02: 05: 33$ & 3.9 & 2.2 & 42.290 & 13.270 \\
197 & 2003 & 7 & 16 & $05: 09: 26$ & 3.2 & 2.6 & 42.330 & 13.280 \\
238 & 2003 & 8 & 26 & $08: 43: 56$ & 3.4 & 2.4 & 42.300 & 13.240 \\
243 & 2003 & 8 & 31 & $10: 33: 00$ & 3.6 & 2.4 & 42.370 & 13.310 \\
239 & 2003 & 8 & 27 & $17: 18: 13$ & 3.6 & 2.4 & 42.520 & 13.27 \\
244 & 2003 & 9 & 1 & $00: 07: 40$ & 3.9 & 2.4 & 42.310 & 13.260 \\
251 & 2003 & 9 & 8 & $23: 03: 50$ & 3 & 2.7 & 42.290 & 13.260 \\
261 & 2003 & 9 & 18 & $21: 00: 40$ & 3.8 & 2.4 & 42.300 & 13.230 \\
267 & 2003 & 9 & 24 & $09: 03: 00$ & 3.2 & 2.8 & 42.480 & 13.230 \\
270 & 2003 & 9 & 27 & $17: 35: 25$ & 4.35 & 3.5 & 42.220 & 13.250 \\
\hline
\end{tabular}
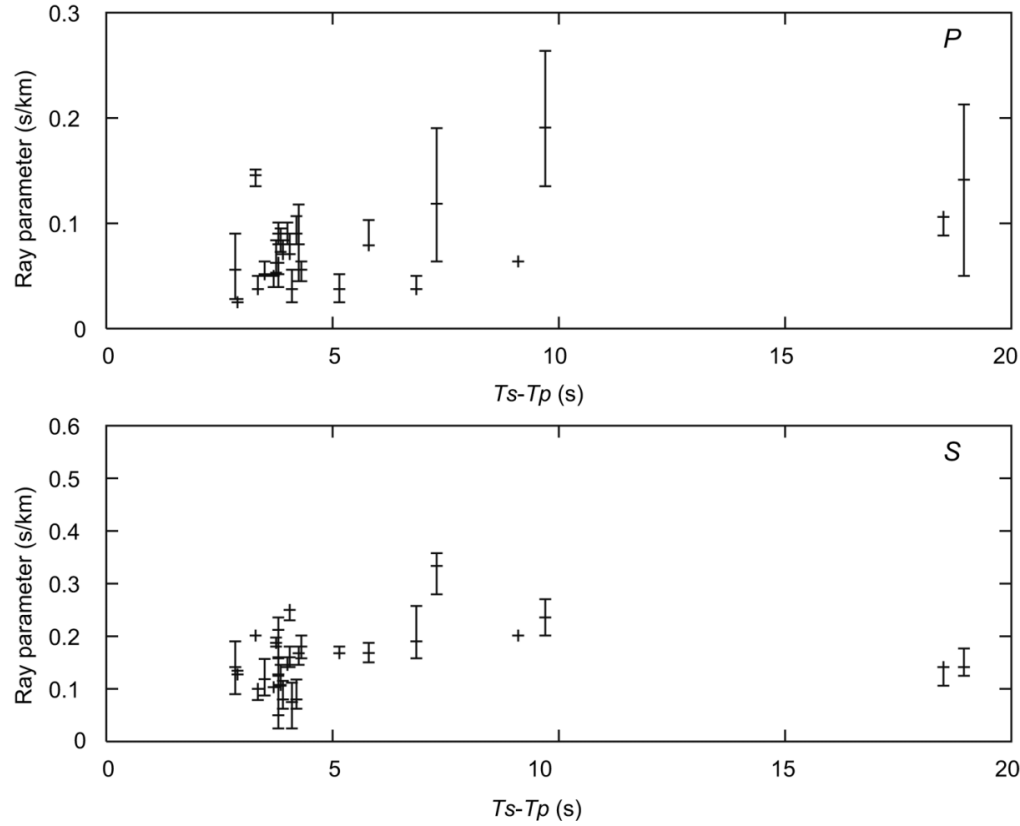

Fig. 11. $P$ - and $S$-waves ray parameters as a function of $S-P$ delay times for all the events listed in table $I$.

errors, these values are compatible with those expected for $P_{n}$ waves. Events located at smaller epicentral distances depict however similar, or even greater apparent velocities, indicating the steep incidence of $P_{g}$ waves from sources whose depth is comparable to the epicentral distance (Braun et al., 2004). As previously mentioned by Biondi and Kostov (1989), ray parameter estimates are much more unstable than the measurement of azimuths. The com- 
parison of $P$ - and $S$-wave ray parameters shows a scattered pattern; however, the estimate of their mutual relationships provides a meaningful value $(1.78 \pm 0.24)$ for the $P$-to- $S$ velocity ra-

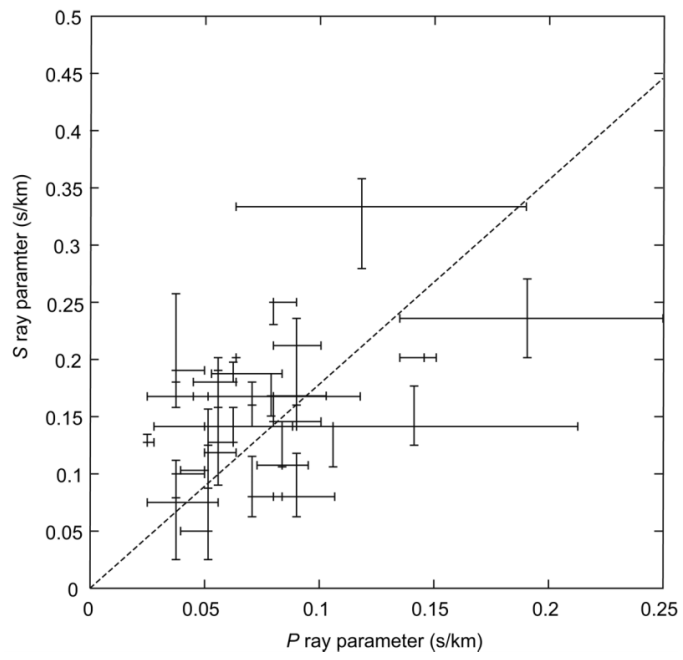

Fig. 12. Comparison of the ray parameters derived from slowness analyses of $P$ - and $S$-waves.

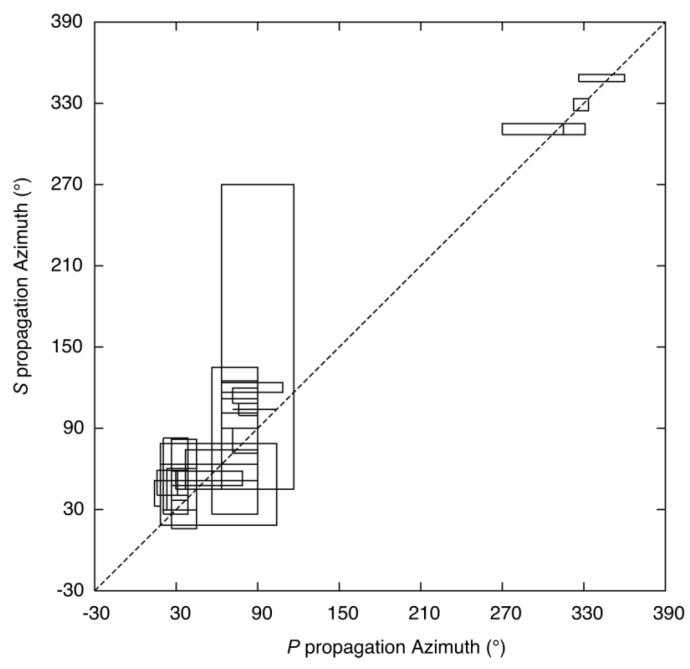

Fig. 13. Comparison of the propagation azimuths derived from slowness analyses of $P$ - and $S$-waves. Width and height of the boxes correspond to the uncertainty on slowness estimates.

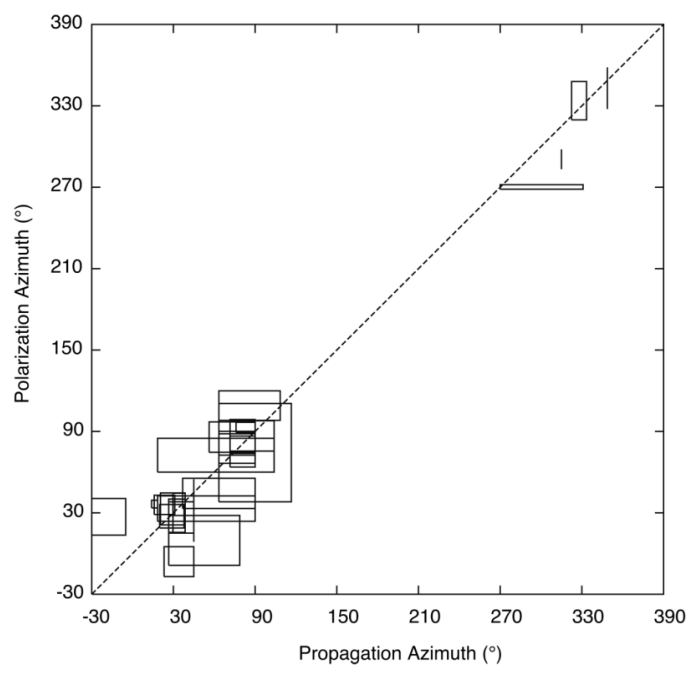

Fig. 14. Comparison of the propagation and polarization azimuths derived from analyses of $P$-waves arrivals. Width and height of the boxes correspond to the uncertainty on slowness and polarization estimates.

tio (fig. 12). Within the error bounds of individual measurements, a general consistency is also observed among the $P$ - and $S$-waves wave propagation azimuths (fig. 13), as well as among the $P$-waves polarization and propagation azimuths (fig. 14). The large uncertainties of slowness estimates observed in figs. 11 and 12 for both $P$ and $S$-waves result from the large width of the main peak in the array response pattern, which is in turn controlled by the array aperture. Thus, major resolution improvements are expected following the enlargement of the array presently under completion.

\section{Location}

At the beginning, we had hoped to obtain consistent estimates on the locations of UNDERSEIS data from back-tracing the seismic wave-vectors evaluated from frequency-slowness analysis (e.g., Almendros et al., 2001). However, due to the large errors associated with ray parameter measurements, and the intrinsic 

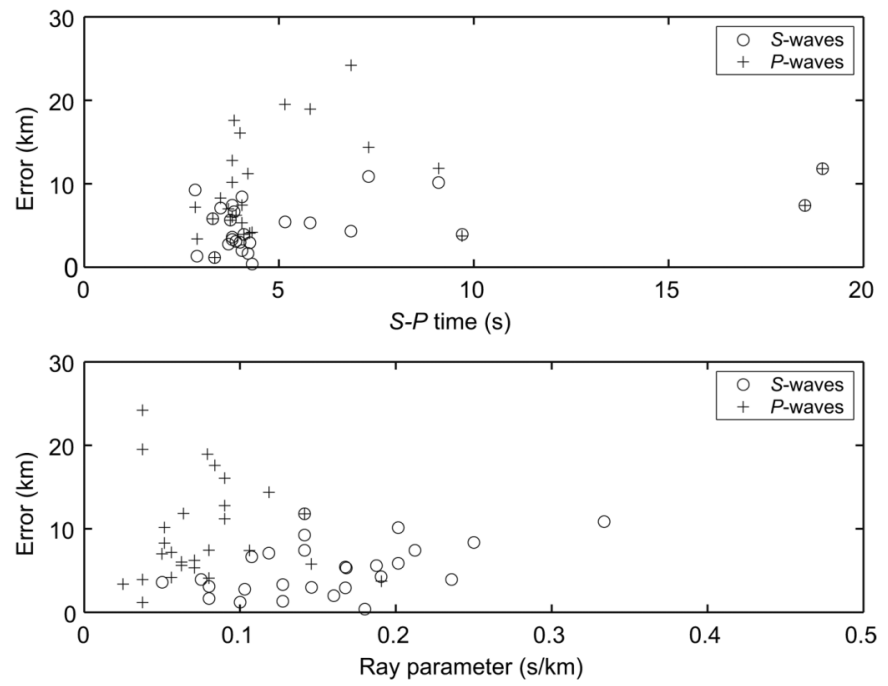

Fig. 15. Location discrepancies as a function of epicentral distance (top) and ray parameter (bottom) from both $P$ - and $S$-waves data.

uncertainties in wave types for events located at ranges comparable to the cross-over distance, this procedure didn't provide satisfactory results. We thus applied a much simpler procedure, deriving epicentral locations from propagation azimuths and using a factor $\gamma$ of 7.5 to convert $S$ - $P$ time delays to epicentral ranges. Error bounds on such locations are conveniently derived from the uncertainties in azimuths estimates, and considering a variation on $\gamma$ of \pm 0.5 . Such uncertainty accounts for errors in $S$ $P$ time delays estimates, and for the fact that the same $S$ - $P$ delay time may be associated with events having different depths and hence different epicentral distance. For all the cases, the epicenters given by the CNSN catalogue are included into the confidence bounds of our estimates. Figure 15 reports the discrepancies among UNDERSEIS and CNSN locations as a function of epicentral distance (top) and ray parameter (bottom) for both $P$ - and $S$-waves. We observe that locations from $S$-waves depict discrepancies which rarely exceed $10 \mathrm{~km}$, and are much more consistent than those retrieved from $P$-wave data. This is quite surprising: one would expect slowness analysis on the $S$-wave to provide less robust estimates, caused by path and recording site effects and by inaccurate sensor orientation. On the other side, however, the amplitude of $S$-waves is generally larger than that of $P$-waves, thus implying significant SNR improvements. Moreover, we have to consider that our slowness spectra were calculated over a cartesian slowness grid of regularlyspaced grid nodes: therefore, slowness measurements become progressively less accurate as one moves toward the origin of the grid, i.e. as the apparent velocity increases. Under such conditions, loss of precision is also induced by round-off errors once deriving very small interstation phase shifts from the eigen-structure of the spatial cross-covariance matrix. Examination of the relationships among location errors and ray parameters shown in fig. 15 confirms these two latter observations. The largest location errors are in fact associated with $P$-wave propagating at low ray parameters, thus suggesting that wave amplitude and velocity are the main factors controlling the precision of slowness measurements.

Keeping in mind these limitations, the above location results may be considered rather satisfactorily, thus indicating that future operations of UNDERSEIS will significantly help to 
better delineate the distribution of micro-seismicity in the Central Apennines.

\section{Discussion and conclusions}

\subsection{Limitations of the array}

The two key factors controlling the accuracy of array-based location procedures are: 1) the precision of slowness measurements, and 2) the ability to correctly predict ray-paths through the region encompassing the array and the epicenter. As for the first factor, in the previous section we observed that the largest location errors were associated with steeply-inciding $P$ waves. This is in agreement with theoretical prediction of slowness uncertainties (e.g., Goldstein and Archuleta, 1991), in which for a given array configuration the robustness of slowness estimates are mostly controlled by SNR and apparent velocity. Thus, slowness measurements derived from the faster-propagating, lower-amplitude $P$-waves are expected to be less accurate than those retrieved from the corresponding $S$ waves arrivals. At the time of revising this paper (early January 2006), 6 additional elements were added to UNDERSEIS, which may now count on a total aperture of about $800 \mathrm{~m}$. Major technological upgrades are also under development in order to allow for sampling rates higher than actually available. From such improvements, we expect a significant increase in measurement precision: on one side, the larger array aperture will improve the resolving capabilities toward fast waves, while on the other the higher number of available stations will reduce the contribution of noise, whose effects are seen on the measurement accuracy scale as $1 / \sqrt{N}$. Regarding the second factor mentioned above, the accurate prediction of ray paths would require knowledge of the velocity structure down to scale lengths in the order of a few hundreds of meters, a task which is obviously beyond the purpose of this study. However, the currently missing assessment of source depths may be attained using gradient velocity models. Such procedure, although producing biased estimates for individual event locations, would preserve the alignments depicted by clusters of adjacent sources; final constraints on absolute locations of these clusters will eventually be gained by joint inversion of array wave-vectors and CNSN arrival-time data associated with the most energetic events.

\subsection{Future perspectives}

In the present study, we were mostly interested in evaluating the location performances of the array: therefore, we selected and analyzed only a subset of the array catalog for which CNSN locations were available. The present efforts are mostly aimed at developing procedures for the automatic detection of earthquake signals, and a prototype on-line processor based on the algorithms presented above is already under testing. Following these efforts, the most immediate goal of UNDERSEIS is to improve the completeness of the seismic catalogue for the Central Apennines. At present, the detection magnitude threshold of the CNSN varies between 1.5 and 2.6 throughout the national territory, and is about 2.2-2.4 for the Central Apennines (Cattaneo et al., 2002). However, from the arguments presented above, we do expect UNDERSEIS to contribute significantly to the detection and location of micro-earthquakes under poor SNR conditions, thus opening the way to the precise definition of seismicity patterns in the surroundings of the array.

In this work, we selected the relevant parameters for slowness measurements (frequency band and length of the time windows) based on sample estimates of the signal's frequency content and coherency. These properties, however, vary significantly from event to event. Any automatic array processing scheme should therefore include the possibility of performing the analysis only over those time-frequency windows for which the signal has maximum energy and coherency (Cornou et al., 2003), in turn adjusting the length of the window of analysis to the frequency band being analysed. This process could be made faster without loss of resolution by adopting sophisticated Time-Frequency decompositions of the signal for the calculation of the frequency-dependent spatial covariance matrices. For this purpose, an extension of the polarization and slow- 
ness measurements to the wavelet-transformed signals (e.g., Bear and Pavlis, 1997) is currently under development. Such improvements would significantly simplify the computational tasks, thus permitting an easier implementation of automatic, on-line detection procedures. Once systematically analysing UNDERSEIS data, a further factor which should be taken into account regards the quantitative definition of the properties of seismic noise, and their variation over time. Although we observed that the noise was spatially uncorrelated, there may be periods of particular meterological condition or intense human activity for which coherent seismic signals may propagate across the array. Future studies must therefore address the problem of a systematic analysis of the characteristics of seismic noise throughout extended time spans, in order to fully elucidate the regions of the slowness space which may be affected by the propagation of coherent signals other than those associated with earthquake sources.

\section{Acknowledgements}

Editor A. Rovelli and reviewers T. Braun and G. Milana provided many thoughtful comments which greatly helped to improving the quality of the manuscript. The financial support from Consorzio Gran Sasso and Laboratori Nazionali del Gran Sasso made possibile the realization of the seismic array. We acknowledge E. Bellotti and E. Boschi for support and stimulation. This work has been conducted in the framework of the program agreement between INGV-Osservatorio Vesuviano and Istituto Nazionale di Fisica Nucleare. We also acknowledge financial support from MIUR-PRIN2005 project «Analisi e modellistica dei processi sismici e deformativi nell'Appennino Centrale».

\section{REFERENCES}

Almendros, J., B.A. Chouet and P.B. Dawson (2001): Spatial extent of a hydrothermal system at Kilauea volcano, Hawaii, determined from array analyses of shallow long-period seismicity, 1. Method, J. Geophys. Res., 106, 13565-13580.

Amoruso, A., L. CRescentini and R. SCARPa (1998): Inver- sion of source paraeters from near-and far-field observations: an application to the 1915 Fucino earthquake, Central Appennines, Italy, J. Geophys. Res., 103, 2998929999.

Amoruso, A., L. Crescentini, A. Morelli and R. ScArpa (2002): Slow rupture of an aseismic fault in a seismogenic region of Central italy, Geophys. Res. Lett., 29, 2.219, doi: 10.129/2002GL016027.

BEAR, L.K. and G.L. PAVLIS (1997): Estimation of slowness vectors and their uncertainties using multi-wavelet seismic array processing, Bull. Seismol. Soc. Am., 87, 755-769

Biondi, B.L. and C. Kostov (1989): High-resolution velocity spectra using eigenstructure methods, Geophysics, $\mathbf{5 4}, 832-842$.

BRATT, S.R. and T.C. BRACE (1985): Locating events with a sparse network of regional arrays, Bull. Seismol. Soc. Am., 78, 780-798.

Braun, T., J. Schweitzer, R.M. Azzara, D. Piccinini, M. CocCo and E. Boschi (2004): Results from the temporary installation of a small aperture seismic array in the Central Apennines and its merits for local event detection and location capabilities, Ann. Geophysics, 47 (5), 1557-1568.

CAPON, J. (1969): High-resolution frequency-wavenumber spectrum analysis, Proc. IEEE, 57, 1408-1418.

Cattaneo, M., P. Augliera and M. de Martin (2002): Seismic noise measurements for the Italian seismic network, Geophys. Res. Abs., SCA/B-3.

Chiarabba, C., A. Amato, P. Augliera, S. Bagh, M. Cattaneo, L. Chiaraluce, P. De Gori, P. Di Bartolomeo, A. Govoni, A. Michelini, M. Moretti, D. Piccinini and M. RoMANELLI (2004): The MAFI project: Mapping Active Faults in Italy using microseismicity data, in Fall Meeting 2004, American Geophysical Union, S52A-01.

Chiarabba, C., L. Jovane and R. Di Stefano (2005): A new view of Italian seismicity using 20 years of instrumental recordings, Tectonophysics, 395, 251-268.

Chiou, S.J. and B.A. Bolt (1993): Seismic wave slownessvector estimation from broad-band array data, Geophys. J. Int., 114, 234-248.

Cornou, C., P.Y. BARD and M. DiETRICH (2003): Contribution of dense array analysis to the identification and quantification of basin-edge-induced waves, Part I. Methodology, Bull. Seismol. Soc. Am., 93, 2604-2623.

Crescentini, L., A. Amoruso and R. Scarpa (1999): Constraints on slow earthquakes dynamics from a swarm in Central Italy, Science, 286, 2132-2134.

De Luca, G., E. Del Pezzo, F. Di Luccio, L. Margheriti, G. MilanA and R. ScARPA (1998): Site response study in Abruzzo (Central Italy): underground array versus surface stations, J. Seismol., 2, 213-226.

De LuCA, G., R. ScArPa, L. FilipPi, A. Gorini, S. MarcuCCi, P. Marsan, G. Milana and E. Zambonelli (2000): A detailed analysis of two seismic sequences in Abruzzo, Central Apennines, Italy, J. Seismol., 4, 1-21.

Goldstein, P. and R.J. Archuleta (1987): Array analysis of seismic signals, Geophys. Res. Lett., 14, 13-16.

Goldstein, P. and R.J. Archuleta (1991): Deterministic frequency-wavenumber methods and direct measurements of rupture propagation during earthquakes using a dense array: theory and methods, J. Geophys. Res., 96, 6173-6185. 
JuRKeVICS, A. (1988): Polarization analysis of three-component array data, Bull. Seismol. Soc. Am., 78, 17251743.

KANASEWICH, E.R. (1981): Time Sequence Analysis in Geophysics (University of Alberta Press, Edmonton), 1-532.

Rost, S. and C. Thomas (2002): Array seismology: methods and applications, Rev. Geophys., 40, 1-27.

Saccorotti, G. and E. Del Pezzo (2000): A probabilistic approach to the inversion of data from a seismic array and its application to volcanic signals, Geophys. J. Int., 143, 249-261.

Scarpa, R., R. Muscente, F. Tronca, C. Fischione, P. Rotella, M. Abril, G. Alguacil, W. De Cesare and M. MARTINI (2004): UNDERSEIS: the UNDERground SEISmic array, Seismol. Res. Lett., 75, 493-504.

SchmidT, R.O. (1986): Multiple emitter location and signal parameter estimation, IEEE Trans. Antennas Propag., 34, 276-280.
Schweitzer, J., J. Fyen, S. MykKeltveit and T. KvaERnA (2002): Chapter 9: Seismic Arrays, in IASPEI New Manual of Seismological Observatory Practice (GeoForschungsZentrum Potsdam), edited by P. BoRMAnN, vol. 1 , pp. 46.

Spudich, P. and D. OppenheImer (1986): Dense seismograph array observations of earthquake rupture dynamics, in Earthquake Source Mechanics, edited by S. DAS, J. Boatwright and C. Scholz, Am. Geophys. Un., Geophys. Monogr. Ser., 37, 285-296.

WANG, H. and M. KAVEH (1985): Coherent signal-subspace processing for the detection and estimation of angles of arrival of multiple wide-band sources, IEEE Trans. AS$S P, \mathbf{3 3}, 823-831$.

(received January 19, 2006; accepted October 20, 2006) 